\title{
RFID Applications in Airline Maintenance Operations
}

Parastoo Dastjerdi ${ }^{5}$

Chris Markou ${ }^{6}$

International Air Transport Association (IATA), Montréal, Canada

Jacques Roy ${ }^{7}$

Department of Logistics and Operations Management at HEC, Montréal, Canada

\section{ABSTRACT}

Radio Frequency Identification (RFID) has been widely used in different industries in recent years but its use in the aviation industry has been very limited. In this article, the use of RFID technology is explored in relationship to airlines' maintenance operations. The main objectives of this article are to assess the current use of RFID in aviation maintenance and to evaluate future opportunities as well as the barriers to this technology in regards to airline maintenance operations. To this end, a survey of airlines was conducted in 2013. The results show that the airline industry has recently taken notice of RFID and that its use is growing. The results also show that airlines are facing several barriers for RFID implementations. They are: lack of knowledge, cost of Enterprise Resource Planning (ERP) integration, cost of tags, lack of support from managers, and immaturity of technology. This research has also identified the categories of parts that can benefit the most from RFID.

${ }^{5}$ Parastoo (Paris) Dastjerdi is a supply chain professional currently working for one of the leading consumer product companies. During the time of this study, she was an employee of IATA and a member of IATA Paperless Operations Task Force. Paris holds a bachelor degree in Industrial Engineering and a Masters in Global Supply Chain Management (HEC Montréal).

${ }^{6}$ Christos (Chris) Markou is Head, Operational Cost Management for IATA. Chris leads the Management and Efficiency programs including the "Paperless Aircraft Operations" initiative to simplify aircraft maintenance. Chris worked at Delta TechOps and holds a Ph.D. (Aerospace Engineering - Georgia Tech) and an Executive MBA (Emory University).

${ }^{7}$ Dr. Roy is a professor at the Department of Logistics and Operations Management at HEC Montreal where he is also Director of the Carrefour logistique, a university-industry forum on Supply Chain Management. He was Director, Research and Publication and Director, Training, at the Montreal based International Aviation Management Training Institute. He holds a Ph.D. (Business administration - HEC Montréal). 
Keywords: Radio Frequency Identification (RFID), Supply Chain Management, Air Transportation Industry, Airline Maintenance, Aviation

\section{INTRODUCTION}

Aviation is an essential part of the global economy. Its worldwide transportation system is the number one enabler of globalization. A recent study of the Air Transport Action Group (ATAG) and Oxford Economics has confirmed that around 56.6 million jobs are supported by aviation globally. In addition, aviation generates about $\$ 2.2$ trillion in global economic activities (ATAG, 2012).

Regardless of all the economic advantages that can be derived from aviation, the average industry profits are not significant and airlines are struggling a great deal in order to recover their cost of capital. Between 1970 and 2010, the average annual post-tax profit for the airline industry has been estimated at about $0.1 \%$ of the annual revenue (IATA, 2013). Moreover, the structure of the airline industry is extremely complex. High value, long-service life and complex configurations are characteristics of capital equipment in the aviation industry, which make managing assets a challenge. Maintaining the aircraft in service for a timeline of thirty plus years is what drives profits (Amann, 2002).

All of these pressures result in constant demand in the market for innovative ideas that provide a competitive advantage. In recent years, airlines have been looking for new innovative ideas that can improve the efficiency of their supply chain management as it is the key to keeping their planes in the air, generating income, and enjoying high customer satisfaction ratings. Radio Frequency Identification (RFID) is an automatic wireless system that has the ability to identify, capture and communicate real-time information in order to facilitate data visibility and product traceability throughout the supply chain. RFID can create improvements in operational activities and result in cost reduction and therefore create a more efficient value chain (Sarac, Absi, \& Dauzère-Pérès, 2010).

The serviceability and maintenance of aircraft is very important for airlines as their operations can shut down without the proper performance of such systems. In addition, the logistics activities of an airline are directly related to the maintenance operations and strongly associated with the competitive advantages of the airline. Considering the challenges in asset and inventory management and the need for innovation to create competitive advantage in airlines, maintenance operations represent a suitable case for assessing the application of RFID.

This research was conducted in collaboration with the International Air Transport Association (IATA). It uses a survey approach to investigate the application of RFID in Airline Maintenance Operations. The main objectives are to assess the current use of RFID in aviation maintenance and evaluate the future opportunities as well as the barriers to this technology in regards to airline maintenance operations. More specifically, the objectives are 1) to explore the status of the industry regarding the use of RFID in maintenance operations, 2) to identify the categories of aircraft parts that can benefit the most from RFID tagging, 3) to provide an example demonstrating the return on investment in maintenance operations, and 4) to discuss the barriers to RFID implementations in maintenance operations and future opportunities. 


\subsection{Literature Review}

RFID systems are made of three main components: the RFID tag, the reader and the communication infrastructure that is called middleware. Middleware acts as a bridge between the RFID and the network database (Ngai, Moon, Riggins, \& Yi, 2008). The idea behind RFID is marking the object with a tag that acts as an identifier and usually has some writable memory to store data. The tag acts on one side as an identifier to locate the object and on the other side to make relevant information about the object available.

In recent years, RFID has been widely used in several industries such as healthcare, fabric and clothing, food, library services, mining and retailing (Ngai et al., 2008). The total market worth of RFID in 2013 was estimated $\$ 7.88$ billion, up from $\$ 6.98$ billion in 2012 . This number is forecasted to increase to $\$ 9.2$ billion in 2014 and $\$ 30.24$ billion in 2024 (Das \& Harrop, 2013). Radio frequency identification (RFID) is believed to be the fastest growing smart label market with an annual estimated growth of $180 \%$ (Ngai et al., 2008).

RFID technology has high potential in the area of aircraft maintenance operations. According to Poirier \& McCollum (2006), the aerospace and defense sectors present the highest possible return for RFID and therefore are the most likely to find acceptance within their industry. US Department of Defense already uses RFID for many parts of its operations. One of which is tracking parts of an airplane. RFID can help facilitate and accelerate parts tracking with the help of real-time information sharing (Poirier \& McCollum, 2006). In 2005, as an initiative to enable airlines to benefit from RFID advantages, the US Federal Aviation Administration (FAA) published a statement that allowed the use of passive RFID on commercial aircrafts. The statement mentioned that passive RFIDs do not cause any harm and safety issues to the aircraft (Chang et al., 2006).

The aircraft manufacturers, Boeing and Airbus, had started using RFID for asset and tool management in the late 1990s. However, in 2006, shortly after the FAA approval of passive RFID, they started planning for RFID solutions and product developments for airline use (Harbison, 2013; O'Connor, 2005). In 2007 Boeing teamed up with Japan Airlines to demonstrate that RFID can speed the inspection process of oxygen generators on board of a Boeing 777 commercial airline (Zaino, 2013). In the same year, a TAP and Airbus team also studied and deployed RFID solution for tracking parts in an engine repair shop (Edwards, 2012). In 2011, Boeing worked with Alaska Airlines on a pilot study to validate the significance of RFID use for labor intensive maintenance (Boeing, 2011). 
Airbus has started tagging life vests and passenger seats across A350 XWB and A320 family, A330, and A380 production lines. In 2009, Airbus took the opportunity to include permanent tags in the aircraft's specifications, covering replaceable parts, Life Limited Parts (LLPs), repairable parts and parts with a Mean Time Between Unscheduled Removals (MTBUR) of less than 60000 hours. Low MTBUR parts are parts that tend to fail more often. Some 2200 components were included for tagging (Harbison, 2013).

Following OEM initiatives, some airlines also started implementing RFID technology for parts management. Delta Air Lines, for example, has been taking advantage of RFID in their maintenance operations. Delta has reported significant time and cost reduction as well as improvements in data accuracy and inventory management because of RFID implementations (Lewis, 2013).

Lufthansa Technik AG (LHT), a leading MRO service provider, has also started using RFID for logistics purposes in order to track the components and parts of the aircraft (Canaday, 2011). In addition, they have started using RFID as an attachment to the documents that travels around with the parts. This allows them to track the movement of the part and the document along the supply chain. The company has reduced manual data entries and the associated errors, and therefore, has improved data accuracy and the speed of the process (Zhang, 2012).

From our literature review, it appears that there are only a few studies on the application of RFID in airline maintenance operations. Despite the recognized advantages that RFID can bring to areas such as inventory management and logistics in airlines, there are very few studies that can be used as a foundation to enquire about the use of RFID in the airline maintenance operations for the purpose of parts management.

\subsection{Methodology}

Following several IATA RFID meetings with a number of airlines and manufacturers, the major airframe OEMs and many airlines suggested having an industry survey conducted to understand the status, perception and needs of the airline industry regarding RFID technology and also to determine what significant aircraft parts could benefit from RFID. A self-administered survey was designed by IATA with the input of two major airframe OEM companies, regulatory authorities and IATA staff. The IATA RFID Survey can be viewed in Appendix 1.

The selected contacts were restricted to airline professionals who were expected to be involved with decisions on using RFID in aircraft maintenance operations. The survey was available to airlines' personnel without discriminating whether the airlines were IATA members or not. This includes engineers, technical and IT professionals, supply chain, and senior management experts closely 
associated with an airline's technical operations (engineering and maintenance) division. In order to capture the individual opinion of experts, it was mentioned that several responses from the same airline would be considered.

The survey was conducted from July 29, 2013 to September 27, 2013.The questionnaire was sent to over 538 individuals at 240 airlines. The airframe OEMs helped disseminating the survey through their customer service departments. At the end of the survey time period, 93 replies were received from 67 airlines. This gave a response rate of 17.3 percent on an individual level and of 28.3 percent on an airline level. For clarification purposes, the Survey was also sent to Approved Maintenance Organizations (AMOs also known as MROs) that are wholly owned or closely affiliated with an airline.

In addition to the RFID survey, interviews, archival data and observations were used. A series of semi-structured interviews were conducted with airline managers who are involved in RFID implementations or have a proven knowledge about the matter. Informal interviews were also conducted when clarifications were needed on certain aspects or answers to the RFID survey.

\subsection{Respondents' Profile}

The highest percentage of respondents belonged to the airlines' engineering department subgroup (40\%). The second subgroup of participants was senior management (27\%) and the third one was maintenance professionals (19\%). Supply chain/logistics (11\%) and other technical professionals $(3 \%)$ were the remaining functions represented on the respondents list. Most of responding airlines were from Europe (42\%), including Russia and the Commonwealth of Independent States (CIS) followed by Asia, Pacific and Oceania (25\%), North America (9\%), Middle East (9\%), Africa (8\%) and South America (7\%). The geographic distribution of our sample reflects the distribution of airlines. More airlines are based in Europe; a highly fragmented market followed by Asia Pacific and Oceania. In terms of size, $31 \%$ of responding airlines have a large fleet of aircraft (greater than or equal to 100$), 40 \%$ have between 30 and 100 planes and $29 \%$ have 30 or less. 


\section{DISCUSSION OF RESULTS}

Not all of the questions were answered by all respondents. Some questions presented a low rate of response because they were not applicable to some respondents. Therefore the sample size for each question is different based on the number of responses received. As applicable, some questions were analyzed at the airline level while others were presented at the individual level since in some cases more than one survey response was received from the same airline.

\subsection{Current Use of RFID in Maintenance Operations}

Out of the 93 individuals who responded to the survey, 19 replied that their airline was implementing RFID at time. These 19 individuals belonged to 12 airlines (18 percent). The remaining 74 individuals from 55 airlines (82 percent) stated that their airline had not implemented any RFID projects yet. A limited number of airlines had embarked in RFID pilot studies a few years ago (2005 - 2008); however these studies were discontinued and were not included as current RFID projects. Table 1 displays the list of region and fleet size of the 12 airlines that are implementing RFID at the moment. As observed in survey results in Table 1, large airlines are predominately exploring the use of RFID along with some medium size airlines. No small airline is working with the technology as of the time the survey was conducted. This is not a surprising result as larger airlines can spread investments over a larger fleet. The benefits from efficiencies in larger fleets are significantly higher. As shown in the cost benefits analysis that was conducted of the use of RFID tags on Oxygen generators (Dastjerdi, 2014), the initial investment increases only slightly with the fleet size. Therefore, airlines with larger fleet size enjoy economies of scale.

Table 1. Airlines with current RFID projects distributed per region and fleet size

\begin{tabular}{|lllll|}
\hline & Fleet $\leq 30$ & $30<$ Fleet $<100$ & Fleet $\geq 100$ & $\underline{\text { Total }}$ \\
\hline Europe & $\mathbf{0}$ & $\mathbf{3}$ & $\mathbf{4}$ & $\mathbf{7}$ \\
\hline North America & $\mathbf{0}$ & $\mathbf{0}$ & $\mathbf{2}$ & $\mathbf{2}$ \\
\hline Asia Pacific \& Oceania & $\mathbf{0}$ & $\mathbf{1}$ & $\mathbf{2}$ & $\mathbf{3}$ \\
\hline Total & $\mathbf{0}$ & $\mathbf{4}$ & $\mathbf{8}$ & $\underline{\mathbf{1 2}}$ \\
\hline
\end{tabular}

Out of the 12 airlines which have current RFID projects, 4 said they implemented RFID on Aircraft (Flyable) parts only, 4 on both aircraft and Non-Aircraft (Non-Flyable), and 4 implemented it on Non-Aircraft parts only. Respondents were also asked to provide the specific parts or group of parts that they were tagging. As shown in Figure 1 of survey results, life vests are the most popular application of RFID in flyable parts with 7 out of the 8 airlines mentioned using RFID for life vests. 
RFID tags are placed on life vests to facilitate and speed up the maintenance checks. While the technician walks through the aisle with a hand-held reader, the status of each vest can automatically be determined by looking at the reader screen. The RFID tag reflects signals to the transmitting reader and would mark any damaged or missing life vests with a red light on the screen indicating absence (loss of signal from the specific life vest). The rest of life vests are given a green light. Therefore, the technician will only check those life vests with a red light, instead of having to manually inspect each and every life vest. Due to pre-synchronization between the life vest and the RFID tag, the technician knows which life vest has the problem (Lewis, 2013).

In addition, RFID is used increasingly for other cabin items. The Industrial Development Director for maintenance components at a major European Airline mentioned using RFID on seat covers and textile at his airline. This allows the airline to track the number of washes for each cover. He explains that some airlines are allowed to perform only a limited number of cleaning per item therefore RFID would be a very useful solution to track the number of washes. He continues: "You have to be able to identify your Part Number (PN) with the textile tag attached to it. If the textile tag (not RFID) is not readable you have to throw away your part, even if some potential cleaning remains and the item is still usable. With the RFID tag, you should be able to identify the good PN and re-issue a proper textile tag to have your item serviceable. In addition, in a warehouse where you have textile it is very tough to do inventory; RFID can provide an easy solution for this problem."

In addition, two major European airlines mentioned using RFID to tag rotables such as engine components. The tags used are low memory and only serve as identification devices for warehouse purposes for now. High memory RFID tags that allow storage of the main history of maintenance of a part are not used for this purpose yet.

Figure 1. RFID activities on aircraft parts

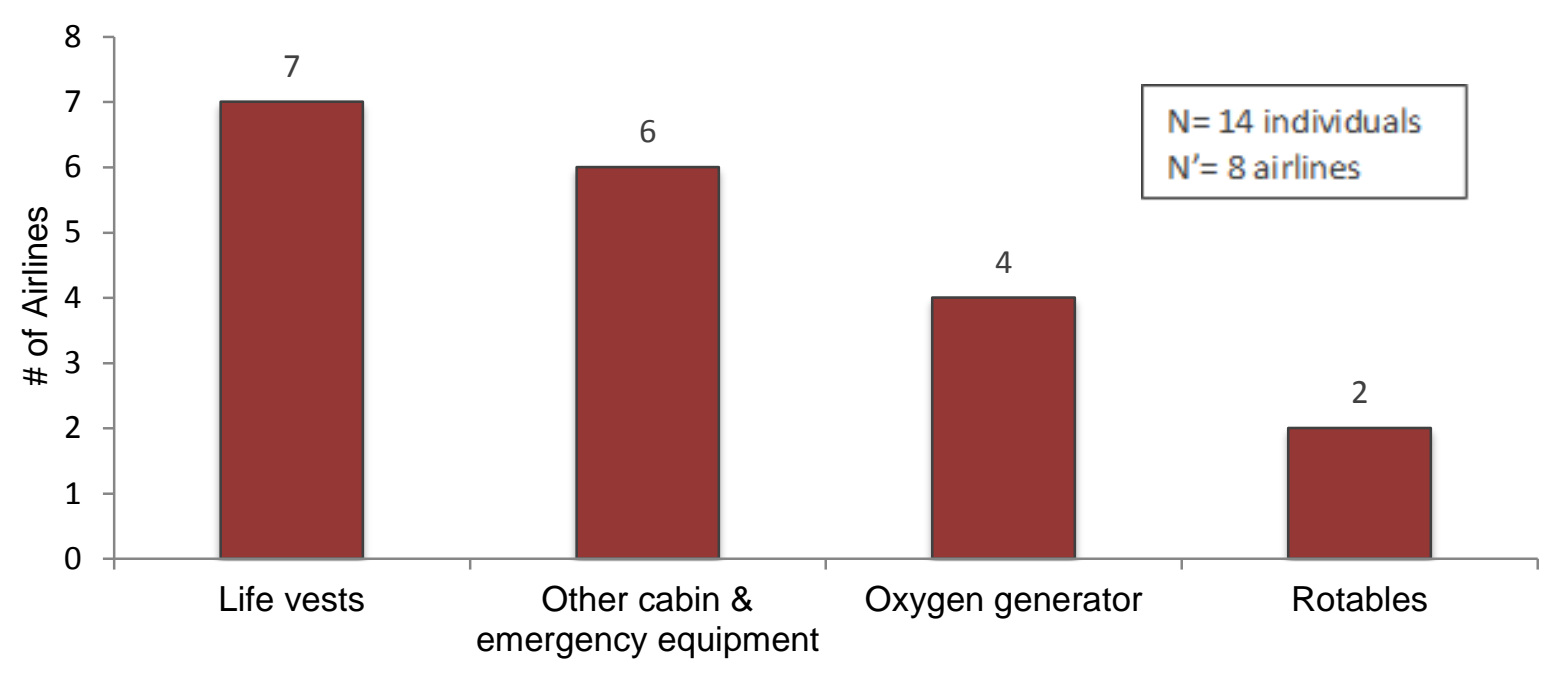


Respondents also provided the specific cases for application of RFID on Non-Aircraft parts. As shown in figure 2, the use of RFID to track tools is a popular application which affects maintenance operations directly. Such uses relate to tool check in/check out by a technician/mechanic, tool location by use of active tags, tools calibration and etcetera. Tools for aviation applications are very specialized and have specific designs that require regular calibration at certain time intervals. In addition, the highly trained aircraft engineers are costly to employ. RFID can minimize the time to look for and to locate tools and allows delegating tasks such as tool calibration to less trained staff (Price, 2007). The "Transportation" category refers to transportation equipment and fleet. In the category "other", shop towels, Employee IDs and Unit Load Devices (ULD) were mentioned.

Figure 2. RFID activities on non-aircraft parts

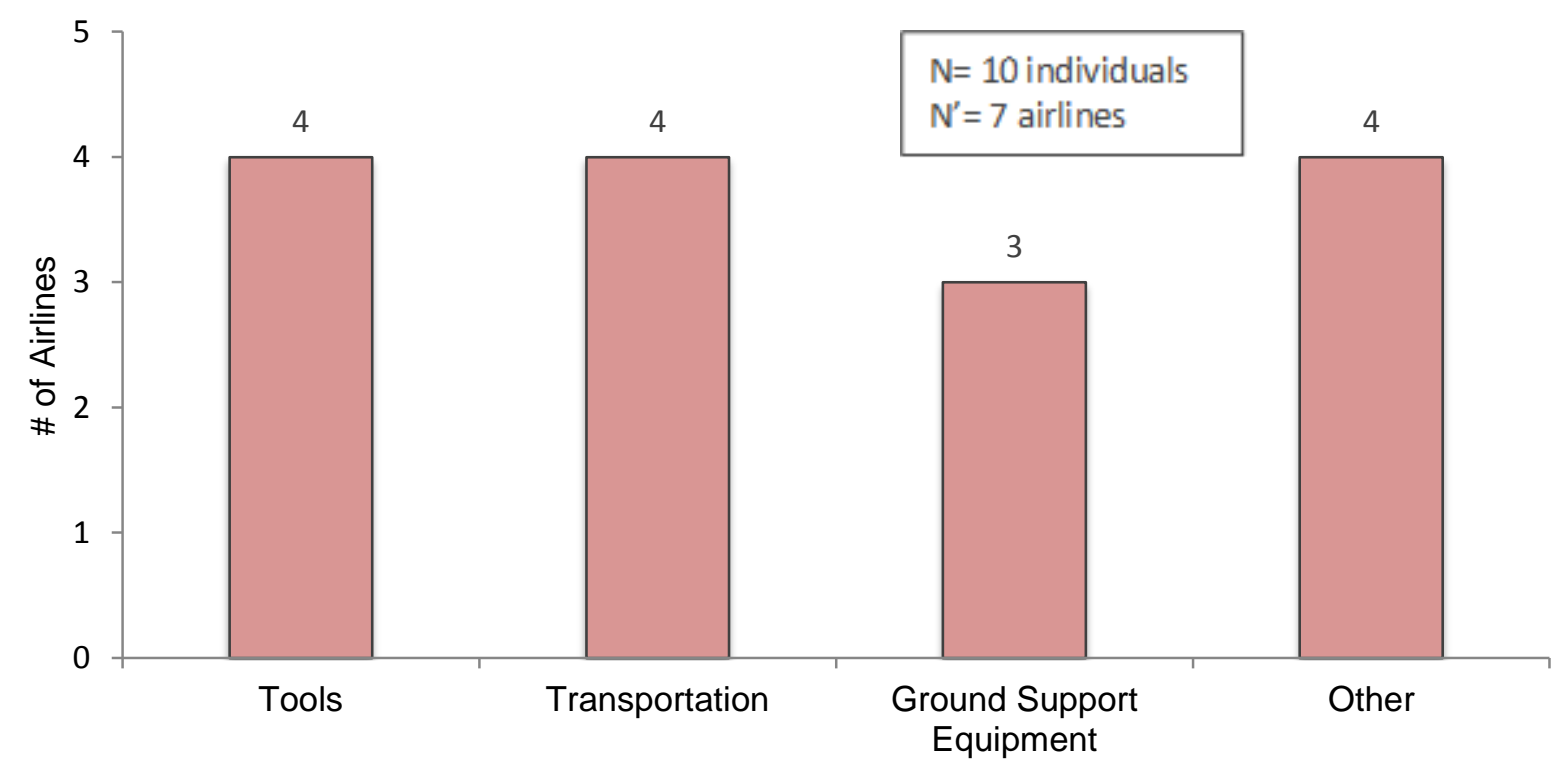

\subsection{Potential Benefits of RFID in Airline Maintenance Operations}

RFID may be an excellent technology but if it doesn't provide a Return on Investment (ROI), its application will be questioned. Airbus has been using RFID for logistics and parts/tools tracking purposes since 2005 and has achieved clear ROI in their RFID applications (Harbison, 2013). Looking at airlines, Lufthansa Technik provides one example of successful RFID implementation to identify and track LLPs in chemical cabinets. Lufthansa has reported "97 percent accuracy and 80 percent time reduction and significant cost savings" (Greengard, 2013). Delta has also seen significant inventory and labor reductions by using RFID (Lewis, 2013). Delta has seen 98 percent time reduction in labor by using RFID to check Oxygen generators (Swedberg, 2013). 
Respondents to our survey were asked to estimate the percentage of improvement they believed RFID brought to their tasks. Although this question is subjective, it shows what each individual perceives regarding the benefits of RFID.

Figure 3 displays the results based on 12 responses from 9 airlines. The missing 3 airlines did not provide an answer because their RFID project has not been fully incorporated yet and therefore this question was not applicable to their situation. The responses circled together come from different individuals within the same airline. Differences between these individuals' perceptions can be explained from the fact that 1) the individuals worked on different projects and 2) the perception was slightly different although showing very close trend.

Figure 3. Improvements upon RFID use ${ }^{8}$

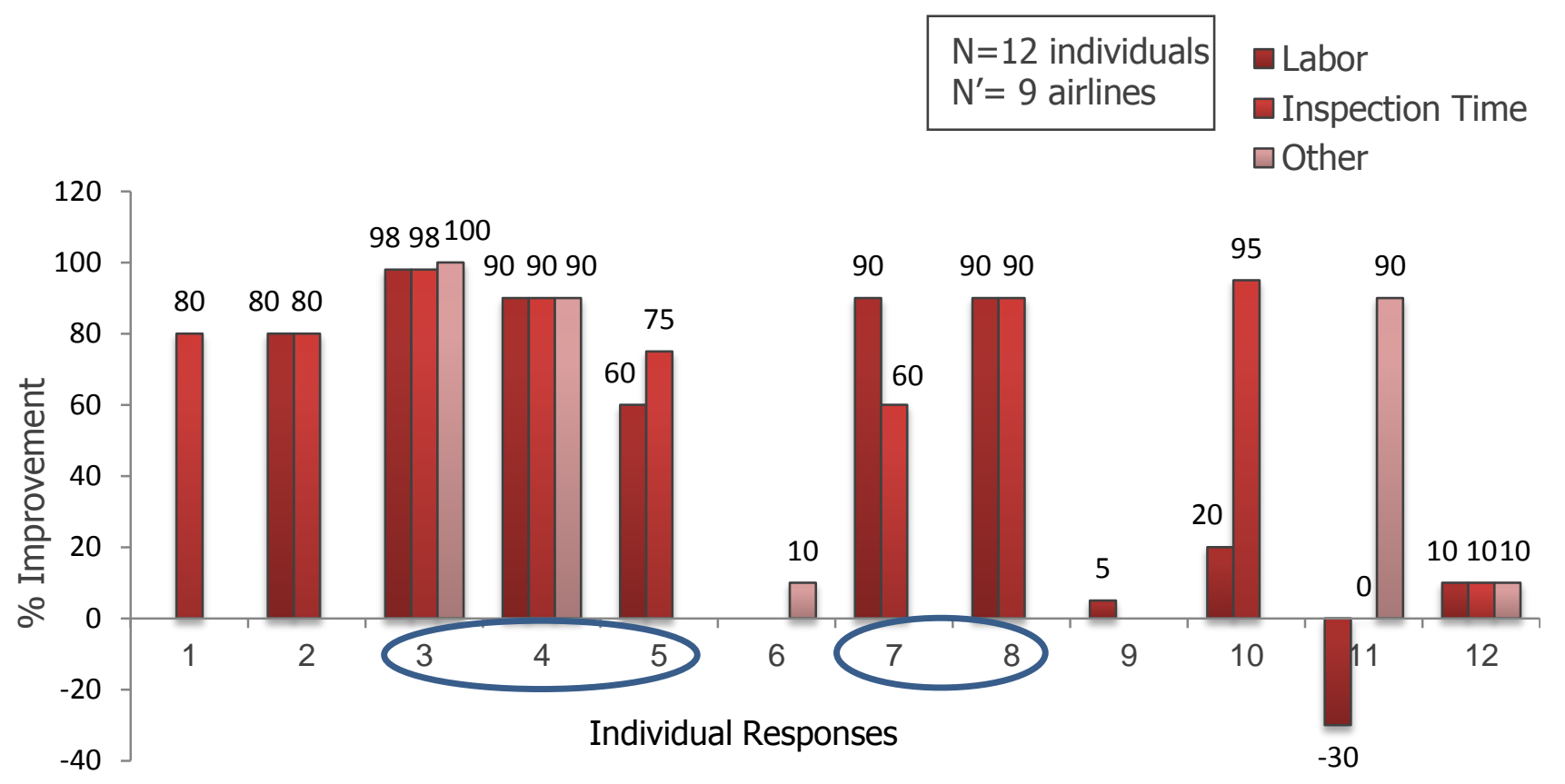

Respondent \#11 reported the implementation of RFID to track shop towels in their engine shop. This was done primarily to improve safety and compliance (i.e. ensuring towels are removed from a component prior to release to service). Although, it has negatively impacted labor productivity by 30 percent, the safety benefits and 100 percent compliance far outweighed the more cumbersome process. Previously, the technicians were free to just grab towels from a bin whenever they needed one. But, after implementing RFID, the technicians had to go through a check-in/check-out process for every shop towel they used. Overall, it was a positive trade-off.

\footnotetext{
${ }^{8}$ The responses circled together come from different individuals within the same airline.
} 


\subsection{Future Use of RFID}

Based on the survey results, current use of RFID application is limited to 18 percent of the industry but this number should be growing as 76 percent responded they are going to have RFID projects in the near future. Out of the 21 respondents from 21 airlines who replied that they were not planning any future RFID projects at the moment, 11 were from small airlines, 4 from medium size airlines and 6 from larger airlines. In order to shed some light on this result, some smaller airlines were interviewed to better understand their perception. The result of the interviews suggested that smaller size airlines normally have a component spare agreement and do not own their own parts; therefore they do not see a reason to use this system. In addition, some mentioned that in smaller airlines it is easier to manage parts manually and there is less need for RFID.

However, being a small airline can be an advantage for RFID adaptation. Literature review confirms that the smaller organization size can make the adaptation of RFID and utilization of the productivity potential easier (Strüker \& Gille, 2010). In addition, occasionally, a carrier may still own or track their assets even if their maintenance is done by a third party on a time and material basis, but per contract, the carrier provides the inventory to the vendor for installation during the repair process (IATA, 2009). In such cases and also for the labor intensive inspections such as checking life vests and oxygen generators, RFID can bring advantage regardless of the size of an airline.

Respondents to our survey also reported on the functions or departments that would gain the most in efficiency improvements through the use of RFID. Results are reported in Figure 4. In the category "other", the following were mentioned: Part pooling, asset tracking and operational health and safety.

Figure 4. Departmental improvements upon RFID deployment

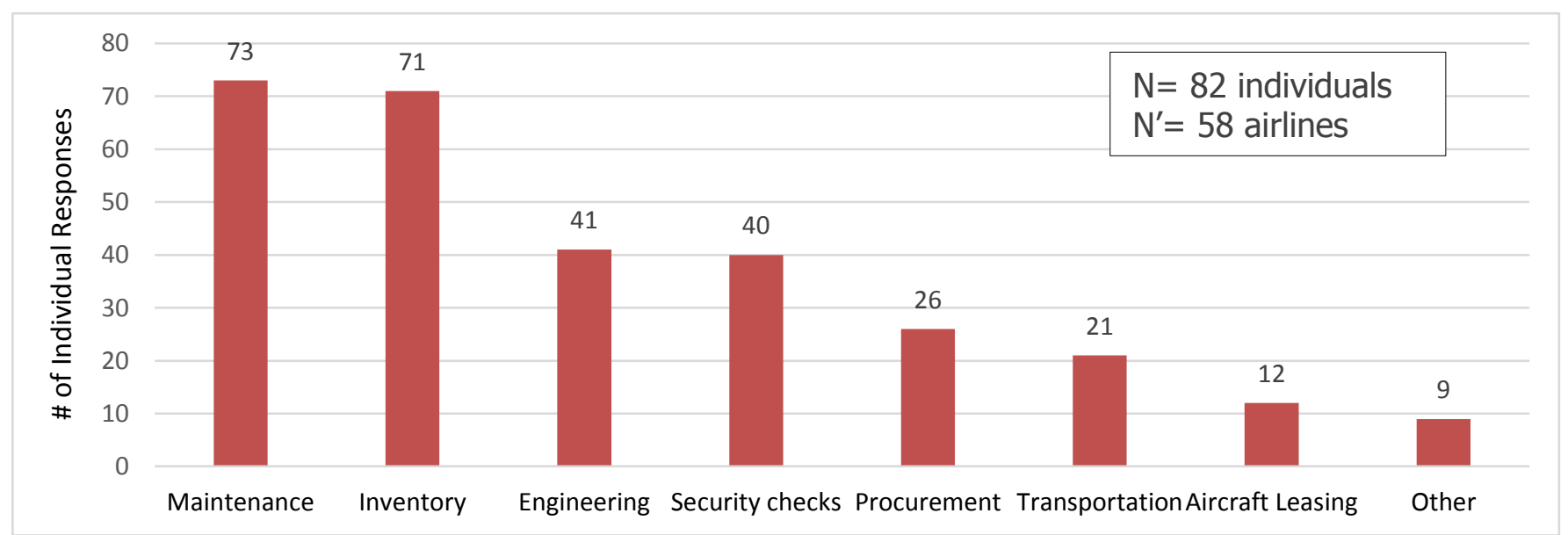


Similarly, survey participants were asked what group of parts were to be given priority for RFID tagging in the future. In other words, which parts could potentially benefit the most from RFID tagging? Results are shown in Figure 5. In the category "other", some respondents mentioned: Tools \& calibrated equipment, Ground Support Equipment (GSE), In Flight Entertainment (IFE) systems, consumables, chemicals and expendables.

\section{Figure 5. Tagging priority}

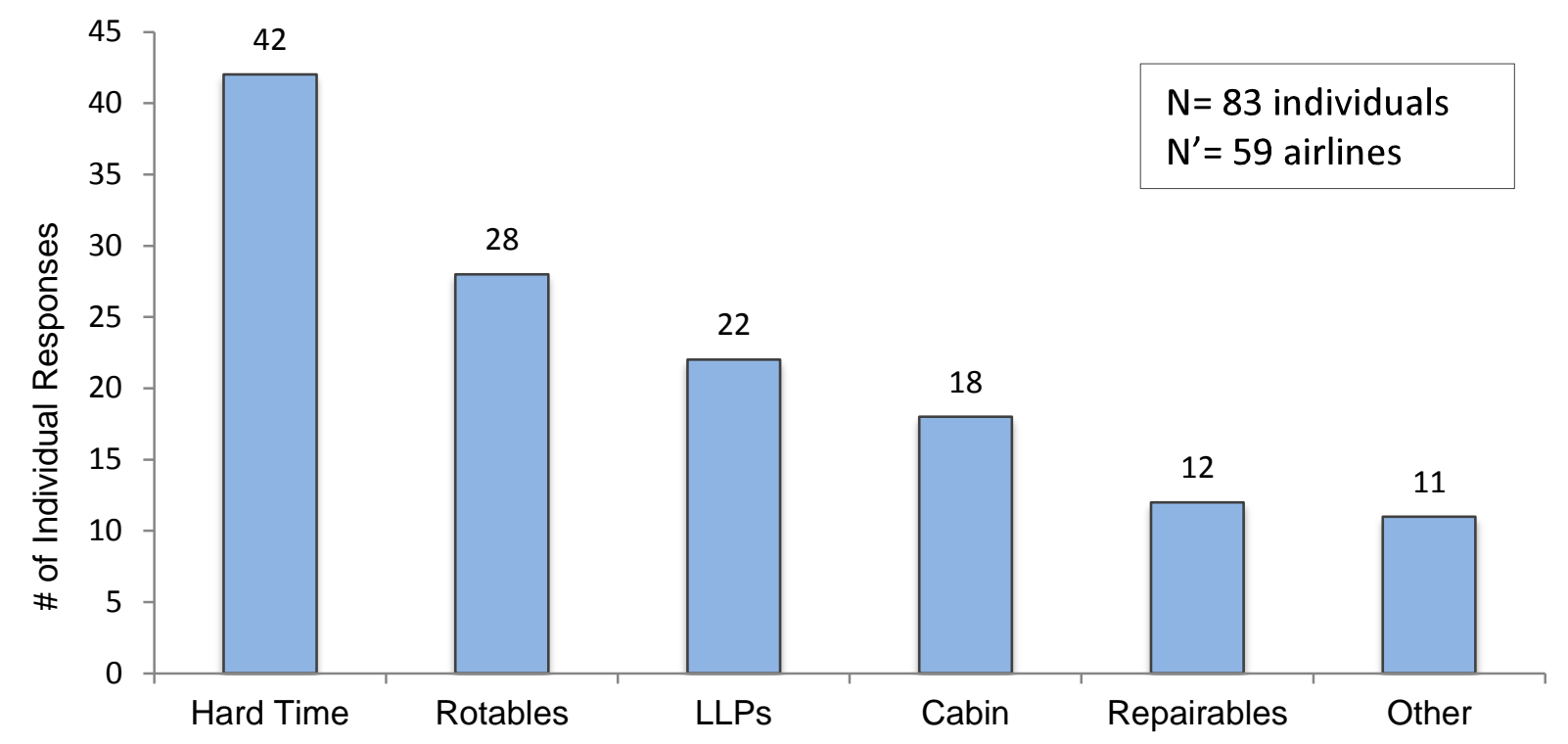

\subsection{Barriers to RFID Implementation}

Respondents were asked to mention the limitations and barriers towards RFID implementation. Lack of knowledge, high cost of ERP interface, high cost of tags, lack of support from senior management, and immaturity of technology were the main barriers according to the respondents as shown in Figure 6.

Figure 6. Barriers to RFID implementation

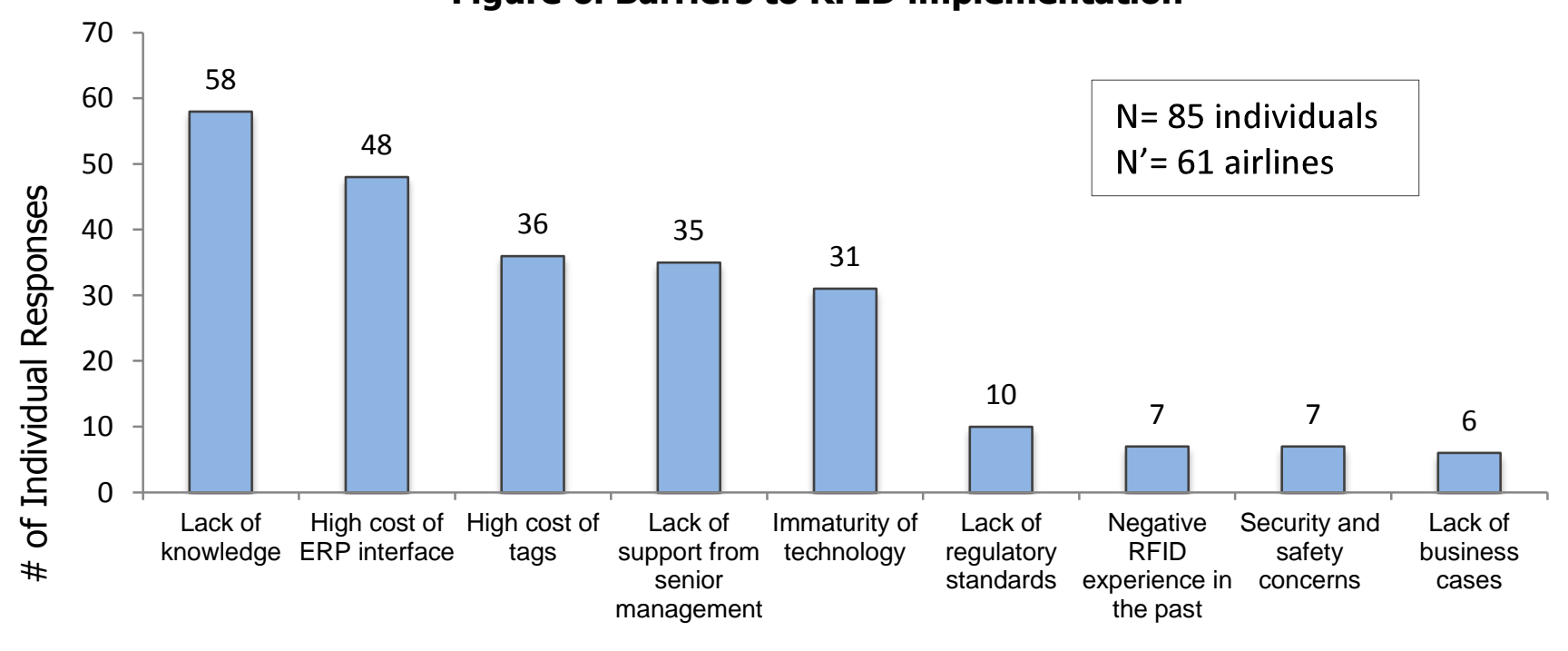




\subsubsection{Lack of Knowledge}

"Lack of knowledge" was identified as the number one barrier. This is visible in the responses that were provided by some respondents as there seems to be a lack of knowledge with respect to some fundamental aspects of RFID. Many respondents replied that it was too difficult to obtain the skills and knowledge necessary to successfully implement a complete system, due to the complexity of configuring and operating numerous hardware and software components. Literature review confirms that potential adopters find it challenging to gain the necessary knowledge and skill for implementation of RFID systems. The configuration and system characteristics are complex and require extensive knowledge (Huang, Qu, Zhang, \& Yang, 2012).

\subsubsection{Cost of ERP Integration}

Respondents also showed significant concerns about the costs of reengineering their processes to support RFID and to integrate the RFID system with their existing information systems. As seen in the literature, the high cost of initial investments in RFID infrastructure may lead to a "wait and see" approach which is the case for many Walmart suppliers. The "wait and see" or "slap-and-ship" approach means tagging the parts right before they leave the supplier site and shipping to the customer. This kind of approach is desirable by some companies as it postpones the need for further investments until the cost of technology decreases (Poirier \& McCollum, 2006).

The application for cabin items such as life vests and oxygen generators does not necessarily need integration with the ERP system. As a major American airline explains, for oxygen generators and life vests, the airline has acquired specific software that connects to the tags and readers and can validate the expiry date and the availability of the vests and generators on the plane. The use of RFID without the full ERP integration would allow airlines to take advantage of the value RFID can offer to some extent without worrying about the investment required in ERP integration.

\subsubsection{Cost of Tags}

Some respondents showed some concern about the high cost of tags. However this concern is mostly attributed to their "lack of knowledge". Today, a tag can cost less than $\$ 1$ US if ordered in quantities. High memory tags can cost more depending on memory size and volume. However, prices are dropping fast and will drop substantially, once the industry firmly commits to the technology. 


\subsubsection{Lack of Support \& Regulatory Standards}

Next on the list, many respondents mentioned a lack of support from senior management. There is also the lack of regulatory standards and limited support from authorities. Some airlines mentioned that there is lack of a real initiative by the type certificate holders and equipment OEMs to create the approvals for items; e.g. where and how the tags should be installed on each part so that it does not interfere in installation or create other system problems. Lack of standards, interfaces with suppliers and MRO ERP systems are also perceived as barriers.

Acceptance of uniform industry standards is required to eliminate complexities and achieve interoperability and higher data security. This is extremely important especially for pool parts; all the operators should be able to read the RFID information. Not many MRO systems vendors have provided RFID interfaces yet. Therefore, one of the main focuses should be on harmonization and standards through the industry in order to facilitate the adaptation of RFID technology. The OEMs should do more than just informing airlines and providing vision and goals. They should also communicate with airlines a detailed implementation timeline that allows airlines to understand the operating factors of RFID as well as the resources required.

\subsubsection{Immaturity of Technology}

Immaturity of technology is another main barrier. Research shows that passive tags do not work well against conductive surfaces. Also hand and arm position affects the read capability (Davis et al., 2010; Griffin et al., 2006; Sydänheimo et al., 2006). In aircraft maintenance, the consequences of tag failure in aircraft maintenance are more severe than it would be in other industries and therefore, this incapability can act as a real burden (Davis et al., 2010).

Some carriers that had or witnessed negative RFID experience were interviewed to gain more insights. As an example, a major Asian airline mentioned that they implemented a pilot RFID project for their oxygen generators in the past but they decided to discontinue. As the airline explained, the main reason for discontinuing at the time was the immaturity of the technology. When a technician was walking through the aisle to scan the generators, if more than one tag was in the neighborhood, the hand terminal reader read the incorrect data.

In addition, sometimes the reader was not able to read the tag. In such cases, the technician had to open the Passenger Service Unit (PSU) in order to access the tag. This resulted in worsening the situation and decreasing work efficiency. It was also mentioned that the reader weighted about 1.4 kilograms and it was heavy for a mechanic to carry. Therefore the airline decided that the maturity of technology was not sufficient, and hence they stopped the implementation. However, it should 
be mentioned that many of the results observed at the time have been already addressed and reversed using current technology.

\subsubsection{Lack of Business Cases \& Other Concerns}

There is significant skepticism around RFID's ability to live up to the performance and capability claims being made by solution providers. Even though solution providers promise great read distances and trouble free implementations, some airlines tend to be quite reserved.

Business cases and industry examples will have to pave the way. One airline mentioned that "it would vastly improve acceptance of the technology if an airline could visit a peer with a working solution and see first-hand that RFID actually works and delivers a quantifiably significant benefit." There are few cases where an airline is acknowledging a vendor's solution. In these cases, some kind of co-marketing agreement could be setup where an airline would be agreeable to help sell an RFID solution in exchange for a share in the profits generated and/or massive discounts. Such arrangements are common for MRO/M\&E software solutions and it does greatly influence a purchase decision to know if a competitor who is using a product is happy with it and is willing to let others see in person why they are so happy with it.

\section{CONCLUSIONS}

The landscape of the aircraft maintenance industry is evolving rapidly. If carriers want to become more competitive or maintain an existing comparative advantage in terms of overall operating costs, they need to be more conscious than ever about maintenance dynamics. RFID can help improve visibility, inventory management, safety and compliance, and speed up processes. These advantages make RFID applications extremely useful and promising for airlines.

Certainly the airline industry has taken notice of RFID, as evidenced by our survey results which showed that 76 percent of the airlines surveyed are planning RFID projects for the future. Comparing this to the 18 percent of airlines surveyed which currently have a RFID project in place, one can see how RFID usage is poised to grow in this industry. All of this indicates that future study and improvements in this area would not only be warranted but welcomed by any organization which is searching for better and quicker ways to do their business.

This work has also identified the category of parts that can benefit the most from RFID. The use of RFID for labor intensive parts such as life vests and oxygen generators is recommended. RFID for tools, ULDs, GSEs and fleet management are other useful applications. This can bring benefits to the maintenance program and facilitate logistics. Depending on the inventory management strategy, 
an airline may also consider application of RFID for other categories such as expendables, parts with low MTBUR, pool components, corrosion monitoring, etc.

Due to some technology barriers, when it comes to conductive surfaces and also due to harsh operating conditions of aircraft, RFID may not be as efficient for tagging engine parts or parts that are in obscure locations or operating under extreme conditions and temperature.

In addition, the barriers to RFID implementations in airlines were identified. Some barriers mentioned are: lack of knowledge, cost of ERP integration, cost of tags, lack of support and regulatory standards, the immaturity of technology, lack of business cases and etc. The number one barrier was identified as the lack of knowledge. Organizations like IATA, have a key role in enhancing the knowledge in the industry. IATA regularly hosts RFID events that encourage experience and knowledge sharing. These events and workshops will have a positive impact on the overall industry knowledge and should be endorsed by airlines' higher management.

Other barriers such as cost of tags and support from authorities and regulatory standards would improve as the technology use becomes more widespread. The growth of RFID use in aviation in the future will contribute to its acceptance and harmonization throughout the airline industry. The immaturity of the technology is another barrier that will be overcome as suppliers will design more sophisticated RFID systems over time. As mentioned previously, there are airlines which have chosen not to use RFID after bad experiences. Improvement in the specificity of the RFID system that eliminates the extra hassles they encountered would be the key to having them adapt to this technology.

Further, as literature review confirms, one of the greatest challenges in RFID research is to bridge the gap between practitioners and researchers (Ngai et al., 2008). This article aimed to give managers and practitioners the type of data and the information that can help them in decision making. Practitioners can take advantage of the results of this article to understand the status and perception of their peer airlines regarding RFID use. The challenges and the benefits associated with RFID implementations identified in this research can also be used as a theoretical model for future implementation projects in the airlines.

\subsection{Study Limitation}

The size of our sample may limit our ability to generalize the results to the whole airline industry. Also, some questions in the questionnaire may not have been clear enough even though the questionnaire was pretested with a few airlines and experts. Therefore, this may have caused confusion for some of the respondents and affected the responses provided. However, in questions 
where possible confusion was suspected, the respondents were individually contacted for clarifications.

\subsection{Future Research}

A literature survey of RFID shows that about a third of all RFID research concentrates on RFID technology and in particular its components (i.e., tags, readers, and antennae). As the technology matures, there should be more attention being paid to less developed research areas, such as business and organizational applications. (Ngai et al., 2008).

That being said and considering the potential of RFID use in the airline industry, more research should be done regarding how best to make use of this technology. Specifically, how can RFID be improved upon to address the current concerns over its applicability. Future studies should also aim at studying the impact of RFID and advantages on the entire aviation supply chain from suppliers to OEMs to MROs to airlines which are the end users.

Further case studies should also be conducted on-site the airlines that are implementing RFID or have previously done so. Such research would focus on the costs and benefits of RFID and determine the areas of improvements needed in order to make the technology use more widespread. 


\section{ACKNOWLEDGEMENTS}

We would like to thank the respondents to our survey and the many industry experts who participated in our research by providing additional information and clarification. We also thank IATA for allowing us to publish the survey results.

\section{REFERENCES}

- Amann, K. (2002). Product lifecycle management: empowering the future of business. 2002, CIM Data: Inc.

- ATAG.

(2012).

Aviation

Benefits

Beyond

Borders. http://aviationbenefitsbeyondborders.org/sites/aviationbenefitsbeyondborders.org/files/pdfs/ABBB_ Medium\%20Res.pdf

- Boeing (Producer). (2011). Boeing, Alaska Airlines Launch Maintenance Cost Saving Program. Retrieved from http://boeing.mediaroom.com/index.php?s=20295\&item=1748

- Canaday, H. (2011). Parts Start Talking. ATW.

- Chang, Y. S., Oh, C. H., Whang, Y. S., Lee, J. J., Kwon, J. A., Kang, M. S., . . . Ung, Y. (2006). Development of RFID enabled aircraft maintenance system. Paper presented at the Industrial Informatics, 2006 IEEE International Conference on.

- Das, R., \& Harrop, P. (2013). RFID Forecasts, Players and Opportunities 2014-2024.

- Dastjerdi, P. (2014) Application of RFID in Airline Maintenance Operation (Unpublished master's thesis). HEC Montréal , Montreal, Canada

- Davis, J., Bowman, D., \& Schmidt, E. (2010). IMPLEMENTATION OF RFID IN AIRCRAFT AND HANGAR MAINTENANCE WITH INVENTORY TRACKING. Advances in Marketing, 41.

- Edwards, J. (2012). Portuguese Airline TAPs Into RFID. RFID Journal.

- Greengard, S. (2013). Lufthansa Technik Automates Supply Management. RFID Journal.

- Griffin, J. D., Durgin, G. D., Haldi, A., \& Kippelen, B. (2006). RF tag antenna performance on various materials using radio link budgets. Antennas and Wireless Propagation Letters, IEEE, 5(1), 247-250.

- Harbison, I. (2013, December). Where are we? MRO Management, 15.

- Huang, G. Q., Qu, T., Zhang, Y., \& Yang, H. (2012). RFID-enabled product-service system for automotive part and accessory manufacturing alliances. International Journal of Production Research, 50(14), 3821-3840.

- IATA. (2009). Guidance Material and Best Practices for Inventory Management: IATA.

- IATA. (2013, 31 December). New Year's Day 2014 marks 100 Years of Commercial Aviation. Press Release No.: 72. Retrieved 27 February, 2014, from http://www.iata.org/pressroom/pr/Pages/201312-30-01.aspx 
- Lewis, R. (2013). Case Study: Delta Airlines leading the way with Inventory Management. Aircraft IT MRO (February-March 2013).

- Ngai, E. W. T., Moon, K. K. L., Riggins, F. J., \& Yi, C. Y. (2008). RFID research: An academic literature review (1995-2005) and future research directions. International Journal of Production Economics, 112(2), 510-520. doi: 10.1016/j.ijpe.2007.05.004

- O'Connor, M. C. (2005). Boeing wants dreamliner parts tagged. RFID Journal.

- Poirier, C. C., \& McCollum, D. (2006). RFID strategic implementation and ROI: a practical roadmap to success. J. Ross Publishing.

- Sarac, A., Absi, N., \& Dauzère-Pérès, S. (2010). A literature review on the impact of RFID technologies on supply chain management. International Journal of Production Economics, 128(1), 77-95.

- Strüker, J., \& Gille, D. (2010). RFID adoption and the role of organisational size. Business Process Management Journal, 16(6), 972-990. doi: http://dx.doi.org/10.1108/14637151011093026

- Swedberg, C. (2013). RFID Reduces Oxygen-Generator Waste for Delta Air Lines RFID Journal.

- Sydänheimo, L., Ukkonen, L., \& Kivikoski, M. (2006). Effects of size and shape of metallic objects on performance of passive radio frequency identification. The International Journal of Advanced Manufacturing Technology, 30(9-10), 897-905.

- Zaino, J. (2013). Boeing Program Automates Aircraft Maintenance Tasks. RFID Journal.

- Zhang, W. (2012). Study on Internet of Things application for High-speed Train Maintenance, Repair and Operation (MRO). 


\section{APPENDIX 1: RFID SURVEY}

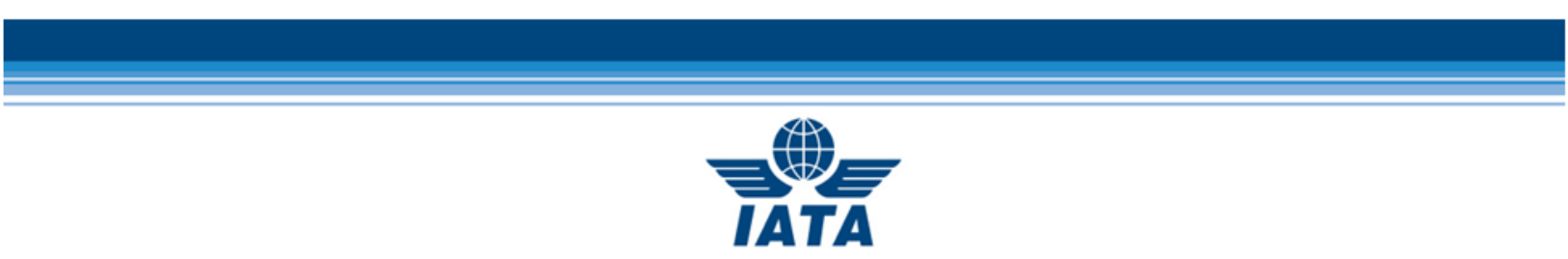

ENGINEERING \& MAINTENANCE

PAPERLESS AIRCRAF T OPERA TIONS

RFID SURVEY 2013

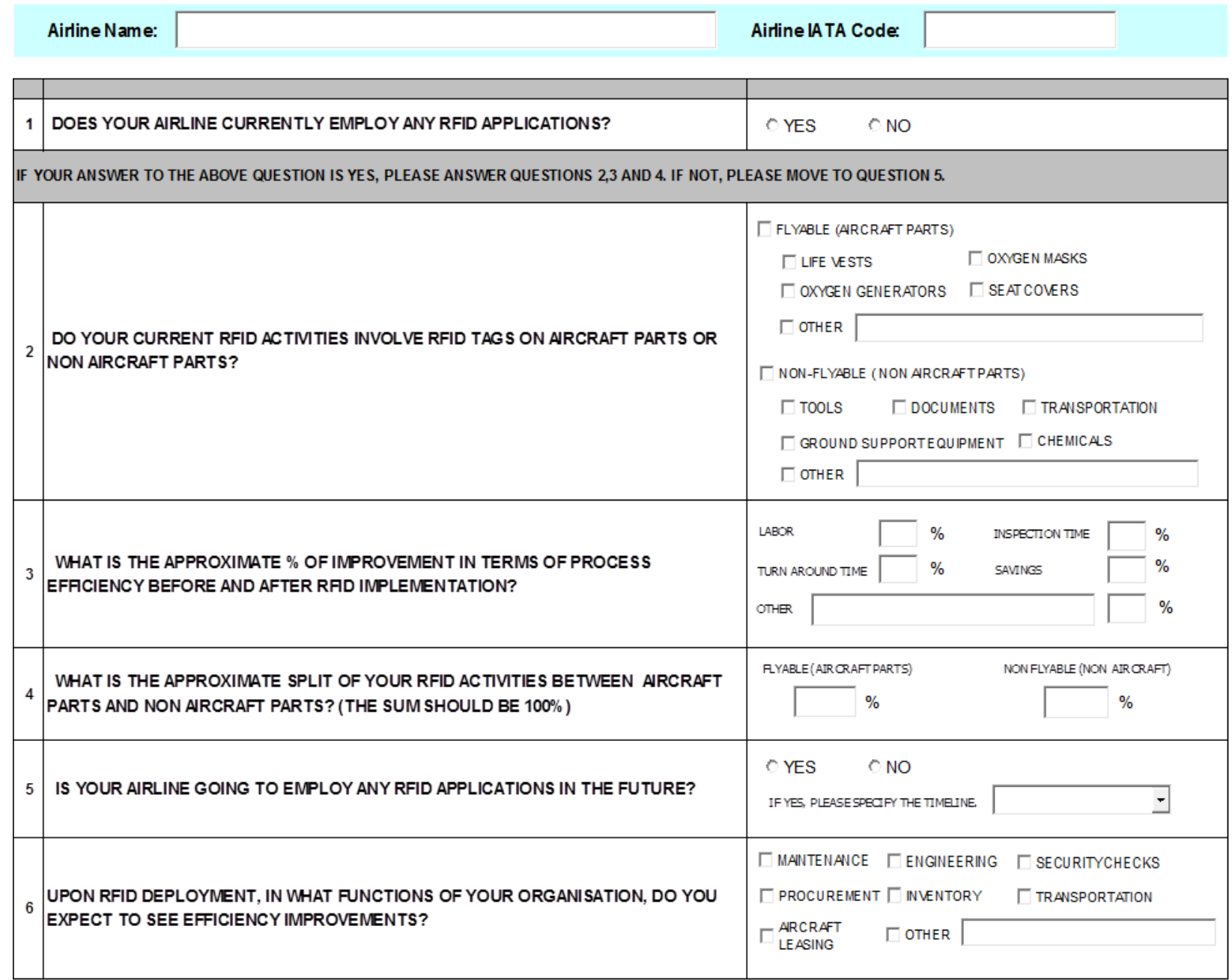




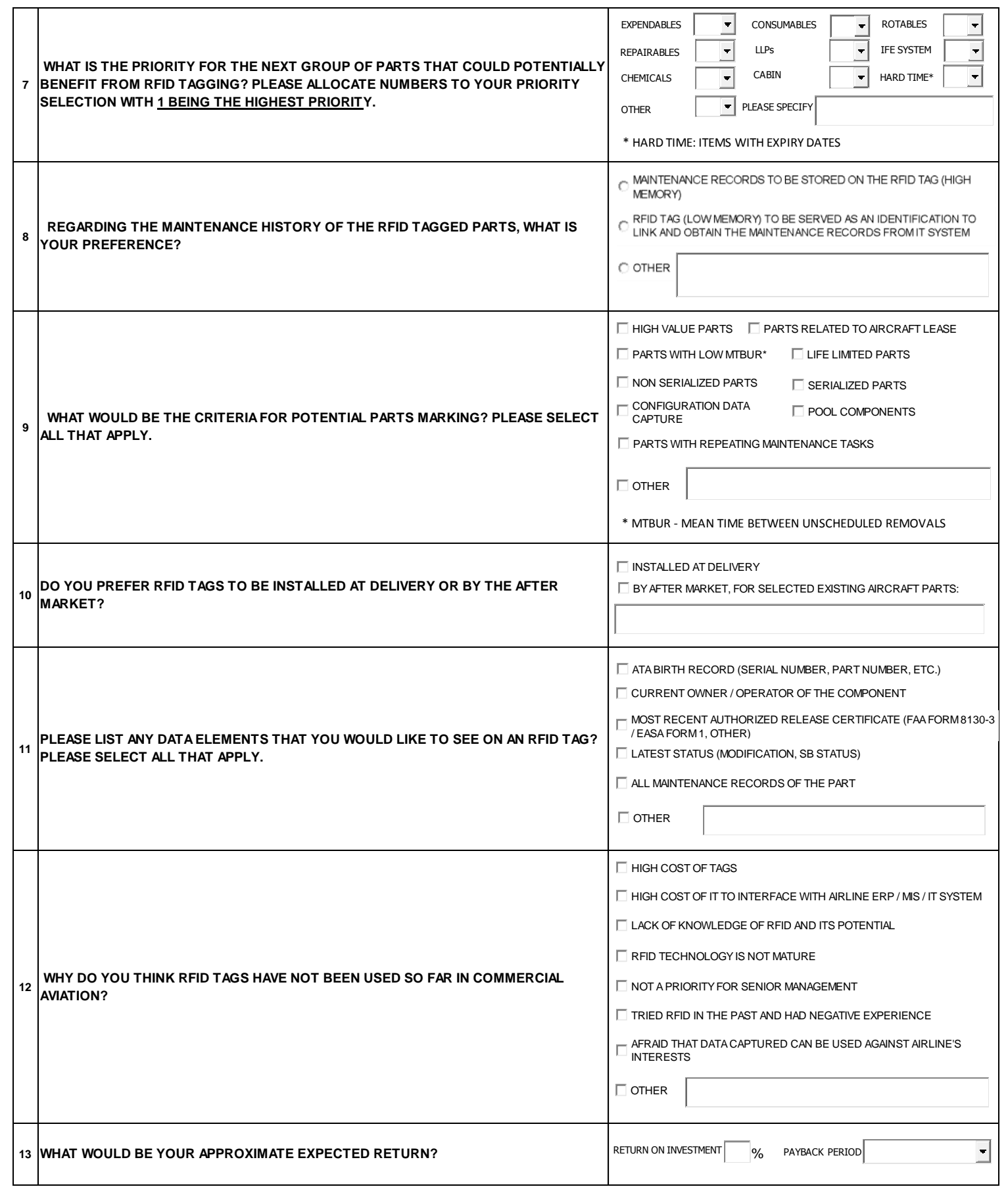

